Article

\title{
Shallow Whole-Genome Sequencing from Plasma Identifies FGFR1 Amplified Breast Cancers and Predicts Overall Survival
}

\author{
Chantal Bourrier ${ }^{1,+}$, Jean-Yves Pierga $2,3,4,+$, Laura Xuereb ${ }^{5}$, Hélène Salaun ${ }^{2,4}$, \\ Charlotte Proudhon ${ }^{3}(\mathbb{D})$, Michael R. Speicher ${ }^{6,7} \mathbb{D}$, Jelena Belic ${ }^{6, \ddagger}$, Ellen Heitzer ${ }^{6,7,8} \mathbb{D}_{\text {, }}$ \\ Brian Paul Lockhart ${ }^{1}$ and Nolwen Guigal-Stephan ${ }^{1, *}$
}

1 Division of Biotechnology, Servier Research Institute, 125, Chemin de ronde, 78290 Croissy Sur-seine, France; chantal.bourrier@servier.com (C.B.); brian.lockhart@servier.com (B.P.L.)

2 Department of Medical Oncology, Institut Curie, 26 rue d’Ulm, 75005 Paris, France;

jean-yves.pierga@curie.fr (J.-Y.P.); salaunhelene@hotmail.fr (H.S.)

3 Circulating Tumor Biomarkers Laboratory, Institut Curie, PSL Research University, INSERM CIC 1428,

26 rue d'Ulm, 75005 Paris, France; charlotte.proudhon@curie.fr

4 Université de Paris, 75005 Paris, France

5 Division of Methodology and Valorisation of Data, Servier Research and Development Institute, 50 rue carnot, 92150 Suresnes, France; laura.xuereb@servier.com

6 Institute of Human Genetics, Diagnostic and Research Center for Molecular BioMedicine, Medical University of Graz, Neue Stiftingtalstrasse 6, 8010 Graz, Austria; michael.speicher@medunigraz.at (M.R.S.); jelena.belic@cruk.cam.ac.uk (J.B.); ellen.heitzer@medunigraz.at (E.H.)

7 BioTechMed-Graz, 8010 Graz, Austria

8 Christian Doppler Laboratory for Liquid Biopsies for Early Detection of Cancer, 8010 Graz, Austria

* Correspondence: nolwen.guigal-stephan@servier.com; Tel.: +33-155-722-532

+ The first two authors have contributed equally to this work.

$\ddagger$ Current address: Cancer Research UK Cambridge Centre, University of Cambridge, Li Ka Shing Centre, Robinson Way, Cambridge CB2 0RE, UK.

Received: 14 April 2020; Accepted: 4 June 2020; Published: 6 June 2020

check for updates

\begin{abstract}
Background: Focal amplification of fibroblast growth factor receptor 1 (FGFR1) defines a subgroup of breast cancers with poor prognosis and high risk of recurrence. We sought to demonstrate the potential of circulating cell-free DNA (cfDNA) analysis to evaluate FGFR1 copy numbers from a cohort of 100 metastatic breast cancer (mBC) patients. Methods: Formalin-fixed paraffin-embedded (FFPE) tissue samples were screened for FGFR1 amplification by FISH, and positive cases were confirmed with a microarray platform (Oncoscan ${ }^{\mathrm{TM}}$ ). Subsequently, cfDNA was evaluated by two approaches, i.e., mFAST-SeqS and shallow whole-genome sequencing (sWGS), to estimate the circulating tumor DNA (ctDNA) allele fraction (AF) and to evaluate the FGFR1 status. Results: Tissue-based analyses identified FGFR1 amplifications in 20/100 tumors. All cases with a ctDNA AF above 3\% $(n=12)$ showed concordance for FGFR1 status between tissue and cfDNA. In one case, we were able to detect a high-level FGFR1 amplification, although the ctDNA AF was below 1\%. Furthermore, high levels of ctDNA indicated an association with unfavorable prognosis based on overall survival. Conclusions: Screening for FGFR1 amplification in ctDNA might represent a viable strategy to identify patients eligible for treatment by FGFR inhibition, and mBC ctDNA levels might be used for the evaluation of prognosis in clinical drug trials.
\end{abstract}

Keywords: clinical trials; FGFR1; liquid biopsy; breast Cancer; sWGS; ctDNA 


\section{Introduction}

A plethora of genomic alterations, including mutations, amplifications, and gene fusions, have been described for the different members of the fibroblast growth factor receptor (FGFR) family in multiple cancer types [1]. In particular, amplification of FGFR1 located at 8p11-12, resulting in overexpression, occurs in less than $10 \%$ across all breast cancer subtypes [2], in approximately 10-16\% of luminal-type breast cancers [3-6], and 4\% in triple-negative breast cancer [7]. FGFR1 amplification and protein expression have been demonstrated as an unfavorable prognosis factor [5,8-10]. Several studies demonstrate that FGFR1 amplifications also play an important role in resistance to endocrine therapy and possibly mediate this resistance mechanism [5]. In addition to ER resistance, FGFR1 amplification has been demonstrated to confer broad resistance to PI3K and CDK4/6 inhibitors [11]. However, despite the clear rationale for targeting FGFR1 in breast cancer with inhibitors, none have, to date, achieved an objective response, and this could be a consequence of inadequate patient selection and/or that FGFR1 may not be the only oncogenic driver in the 8p11-12 amplicon.

In oncology clinical trials, one of the most important challenges is to ensure that the molecular status of the tumor accurately reflects the targeted population at the time of study inclusion. However, in clinical trials, it is sometimes difficult to obtain a fresh tissue biopsy at patient inclusion, especially in phase I trials and for exploratory research (for both ethical and/or medical health reasons). In addition, genomic anomalies present at relatively low to intermediate prevalence require extensive screening of patients. Consequently, molecular analyses, including mutation and somatic copy number alterations (SCNA) characterization, are typically performed on archival tissue, in most cases, acquired through a biopsy at diagnosis, which can significantly pre-date patient inclusion in clinical trials. Moreover, a single tissue biopsy (often a needle biopsy) is unlikely to reflect the global molecular, spatial, and temporal tumor dynamics with regard to both intra- and inter-tumoral heterogeneity, making clinical decisions-based on archival tissue biopsy—challenging.

Although less documented than SNV variation, several studies have also demonstrated both spatial and temporal evolution of gene amplification in cancer progression (in situ to invasive transition), from primary to metastatic tumors or under selective treatment pressure. The frequency of FGFR1 amplification has been shown to be higher in invasive breast carcinomas and in the invasive components of tumors with both invasive and ductal carcinomas in situ (DCIS) components compared to pure DCIS [9]. De novo amplification of FGFR1 has also been detected in a metastatic deposit of breast cancer (BC) patients not present in the primary tumor [12]. Likewise, HER2 status might differ between primary and metastases tumors, and HER2 amplification has been shown to be 'acquired' in about $2-3 \%$ of metastatic breast cancers, following neo-adjuvant chemotherapy treatment [13].

Consequently, based on the dynamics of cancer progression and significant intra-tumor heterogeneity, it is essential to evaluate the molecular profile at patient inclusion rather than relying exclusively on archival tissue. Detection of somatic alterations in cell-free DNA from plasma (cfDNA), which in cancer patients contains tumor-derived DNA fragments (ctDNA, circulating tumor DNA), is already in use in clinical practice and provides valuable information for patient management [14]. SCNA detection in plasma cfDNA by either digital droplet PCR or targeted next-generation sequencing (NGS) is much more challenging and not always cost-effective as mutation analysis. In this context, shallow whole-genome sequencing (sWGS) allows the identification of both genomic structural anomalies and ctDNA tumor fractions in plasma by measuring copy numbers from sequence read depth. This low-cost method has been successfully applied in several studies to monitor treatment response, to identify resistance mechanisms, or to estimate prognosis [15-20]. In this study, we used sWGS to assess the potential use of ctDNA as a surrogate for FGFR1 amplification from metastatic breast cancer patients. In addition, we triaged patients based on their tumor content using two untargeted methods and correlated the basal ctDNA levels with patient survival. 


\section{Results}

\subsection{Characterization of the Cohort and Identification of FGFR1 Amplified Breast Cancer Cases}

Detailed clinicopathological characteristics of the metastatic breast cancer $(\mathrm{mBC})$ cohort $(\mathrm{n}=100)$, covering different subtypes, are described in Table 1. A total of 88 tumors were estrogen receptor-positive $(\mathrm{ER}+)(88 \%)$, of which 60 tumors also demonstrated progesterone receptor positivity $(\mathrm{ER}+\mathrm{PR}+)(60 \%)$. $\mathrm{PR}+$ alone also was observed in only one tumor. Eleven tumors were triple-negative. A total of 88 (92\%) tumors were primary grade 2 and 3 tumors. The median follow-up was 72 months, and the median overall survival was 44 months. As previously reported, FGFR1 [4] and triple-negative [21] tumors were associated with significantly worse survival (Kaplan-Meier log-rank $p=0.01$; Supplementary data Figures S1 and S2) compared to hormone receptor-positive cases, with a shorter disease-free interval of less than 36 months ( $\log$-rank $p=0.035$, Supplementary data Figure S3).

Table 1. Clinicopathological characteristics of metastatic breast cancer cohort. ${ }^{*} 4$ missing data ** 1 missing data. TNBC: triple-negative breast cancer, ER: estrogen receptor, PR: progesterone receptor, DFI: disease-free interval (between primary and distant metastasis diagnosis), IDC: invasive ductal carcinoma, FGFR1: fibroblast growth factor receptor 1.

\begin{tabular}{cccc}
\hline \multirow{2}{*}{ Caption } & FGFR1 Amplified & FGFR1 Amplified & Total \\
\cline { 2 - 4 } & $\mathbf{N = \mathbf { 2 0 }}$ & $\mathbf{N = \mathbf { 8 0 }}$ & $\mathbf{N = \mathbf { 1 0 0 }}$ \\
\hline AGE (years) median & $56(28-78)$ & $60(32-83)$ & $60(28-83)$ \\
$<50$ year & $4 / 20$ & $25 / 80$ & 29 \\
DFI (months) median & $35(0-183)$ & $31(0-227)$ & $33(0-227)$ \\
IDC & $18(90 \%)$ & $71(89 \%)$ & 89 \\
Lobular & $2(10 \%)$ & $9(11 \%)$ & 11 \\
Primary tumor grade & - & - & - \\
1 & 0 & 8 & 8 \\
2 & 9 & 32 & 41 \\
3 & 11 & 36 & 47 \\
ER+/- & $19 / 1$ & $69 / 10$ & $68 / 11$ \\
PR+/- & $13 / 7$ & $47 / 27$ & $60 / 34$ \\
TNBC ** & 1 & $10($ out of 79$)$ & $11($ out of 99$)$ \\
Visceral metastasis y/n & $15 / 5$ & $40 / 40$ & $55 / 45$ \\
Metastatic sites $>2 \mathrm{y} / \mathrm{n}$ & $10 / 10$ & $23 / 57$ & $33 / 67$ \\
\hline
\end{tabular}

Using FISH, FGFR1 amplification was identified in 20 cases (20\%). Of those, 17 cases were classified as amplified and three cases as low amplified based on the criteria defined by Schildauss et al. [22] (Table 2 and Supplementary data Table S1). The FGFR1/CEN8 ratio ranged from 1.1-8.4, with an average number of FGFR1 gene signals per tumor cell of 4.3 ranging from 4.8-25.1. Among the 20 amplified FGFR 1 cases, 13 cases were ER+/PR+, 6 cases were ER+/PR-, and only one case was triple negative. 
Table 2. FGFR1 amplified cases based on FISH testing of a metastatic breast cancer cohort $(n=100)$. * FGFR1 amplification was based on the criteria of Schildhaus et al., 2012. Notably: FGFR1/CEN8 ratio was $\geq 2.0$, or the average number of FGFR1 signals per tumor cell nucleus was $\geq 6$. Additional evaluation criteria (FGFR1 low amplification) were considered if the $\%$ of tumor cells containing $>5$ gene signals was $>50 \%$ and/or $\%$ of tumor cells containing $>15$ gene signals or large clusters was $>10 \%$.

\begin{tabular}{|c|c|c|c|c|c|c|c|}
\hline \multirow{2}{*}{$\begin{array}{l}\text { Caption } \\
\begin{array}{c}\text { Case No. } \\
\text { (Patient } \\
\text { ID) }\end{array}\end{array}$} & \multicolumn{4}{|c|}{$\begin{array}{c}\text { Evaluation Criteria * } \\
\text { FGFR1 was Amplified, If } F G F R 1 / C E N 8 \text { Ratio was } \geq 2.0 \\
\text { and/or } \\
\text { Average Number of } F G F R 1 \text { Signals/Tumor Cell was } \geq 6.0\end{array}$} & \multicolumn{3}{|c|}{$\begin{array}{c}\text { Additional Evaluation Criteria (Low Amplification) } \\
\text { FGFR1 was Amplified, If the Percentage of Tumor Cells } \\
\text { Containing } \geq 5 \text { Gene Signals was } \geq 50 \% \\
\text { and/or } \\
\text { Percentage of Tumor Cells Containing } \geq 15 \text { Gene Signals or } \\
\text { Large Clusters was } \geq 10 \%\end{array}$} \\
\hline & $\begin{array}{c}\text { Target } \\
\text { Gene/Centromere } \\
\text { Ratio }\end{array}$ & $\begin{array}{l}\text { Average Number } \\
\text { of Target Gene } \\
\text { Signals/Nucleus }\end{array}$ & $\begin{array}{l}\text { Average Number } \\
\text { of Centromere } \\
\text { Signals/Nucleus }\end{array}$ & $\begin{array}{c}\text { FGFR1 } \\
\text { Status }\end{array}$ & $\begin{array}{c}\text { Percentage of Tumor } \\
\text { Cells Containing } \geq 5 \\
\text { Gene Signals (Quotient) }\end{array}$ & $\begin{array}{l}\text { Percentage of Tumor } \\
\text { Cells Containing } \geq 15 \\
\text { Gene Signals or Large } \\
\text { Clusters(Quotient) }\end{array}$ & $\begin{array}{c}\text { FGFR1 } \\
\text { Status }\end{array}$ \\
\hline 525973 & 1.9 & 7.7 & 4.1 & amplified & 0.9 & 0.1 & amplified \\
\hline 529014 & 3.4 & 7.2 & 2.1 & amplified & 0.9 & 0 & amplified \\
\hline 531084 & 2.5 & 6.3 & 2.5 & amplified & 0.8 & 0 & amplified \\
\hline 537264 & 1.1 & 7.5 & 7.1 & amplified & 0.9 & 0.1 & amplified \\
\hline 542827 & 4.5 & 11.6 & 2.6 & amplified & 1.0 & 0.3 & amplified \\
\hline 548602 & 6.7 & 16.2 & 2.4 & amplified & 1.0 & 0.6 & amplified \\
\hline 595763 & 2.5 & 10.5 & 4.2 & amplified & 1.0 & 0.1 & amplified \\
\hline 599471 & 5.4 & 9.8 & 1.8 & amplified & 1.0 & 0.1 & amplified \\
\hline 600575 & 6.2 & 12.8 & 2.0 & amplified & 1.0 & 0.4 & amplified \\
\hline 615064 & 1.9 & 4.8 & 2.6 & balanced & 0.6 & 0 & amplified \\
\hline 621823 & 5.6 & 12.7 & 2.2 & amplified & 1.0 & 0.4 & amplified \\
\hline 623854 & 4.6 & 7.8 & 1.7 & amplified & 0.7 & 0.1 & amplified \\
\hline 630510 & 4.2 & 5.3 & 1.3 & amplified & 0.6 & 0 & amplified \\
\hline 631774 & 1.9 & 5.1 & 2.7 & balanced & 0.6 & 0 & amplified \\
\hline
\end{tabular}

As a next step, whole-genome copy number analysis using the Oncoscan ${ }^{\mathrm{TM}}$ technology was used as orthogonal confirmatory testing for FGFR1 amplified cases. Out of the 20 FGFR1 amplification positive tumors identified with FISH, only 11 had sufficient amounts of DNA for the array analysis with no low amplified cases present (Table 3). With the exception of one case (N\# 542827), Oncoscan confirmed the presence of FGFR1 amplification, resulting in a concordance rate of $91 \%$. Despite this high consistency in FGFR1 amplification status between both technologies, the level of amplification only showed a weak correlation $(r=0.399)$ (Supplementary data Figure S4). At univariate analysis, FGFR1 amplification was associated with shorter overall survival (OS) (median 32 months versus 54 months, $\log$-rank $p=0.0018$ ). During multivariate analysis, independent pejorative factors for OS were FGFR1 amplification, triple-negative status, and a shorter disease-free interval (DFI).

Table 3. FGFR1 amplification status concordance between FISH and Oncoscan testing.

\begin{tabular}{cccccc}
\hline $\begin{array}{c}\text { Case No. } \\
\text { (Patient } \\
\text { ID) }\end{array}$ & $\begin{array}{c}\text { FGFR1 } \\
\text { Status } \\
\text { Oncoscan }\end{array}$ & $\begin{array}{c}\text { FGFR1 Copy } \\
\text { Number } \\
\text { Oncoscan }\end{array}$ & $\begin{array}{c}\text { Concordance } \\
\text { with FISH }\end{array}$ & Biopsies-FISH & $\begin{array}{c}\text { Copy Number: Average } \\
\text { Number of Target Gene } \\
\text { Signals/Nucleus }\end{array}$ \\
\hline 529014 & Gain & 3 & Yes & Amplified & 7.2 \\
531084 & Gain & 4 & Yes & Amplified & 6.3 \\
542827 & No gain & 2 & No & Amplified & 11.6 \\
548602 & Gain & 5 & Yes & Amplified & 16.2 \\
557513 & Gain & 8 & Yes & Amplified & 10.8 \\
562315 & Gain & 24 & Yes & Amplified & 25.1 \\
587002 & Gain & 5 & Yes & Amplified & 15.6 \\
594270 & Gain & 16 & Yes & Amplified & 11.6 \\
600575 & Gain & 7 & Yes & Amplified & 12.8 \\
623854 & Gain & 15 & Yes & Amplified & 7.8 \\
630510 & Gain & 5 & Yes & Amplified & 5.3 \\
\hline
\end{tabular}




\subsection{SCNA Analysis in cfDNA and Evaluation of the Tumor Level}

For ten of the initial 20 FGFR1 amplified cases, corresponding plasma was available. In addition, 10 further cases without FGFR1 amplification were randomly selected and analyzed as controls. cfDNA was initially analyzed using a modified fast aneuploidy screening test-sequencing system (mFAST-SeqS), which is basically a PCR with one primer pair that amplifies $>20000$ different LINE-1 sequences. The amplification product enables the calculation of a genome-wide z-score, which reflects aneuploidy and hence the level of ctDNA present in cfDNA [23]. For these 20 samples, the genome-wide z-score ranged from 1.1 to 31.5, with a median of 2.2. Only eight samples had a z-score greater than three, which was previously used as a threshold for further processing with sWGS (Figure 1 and Table 4). Since the resolution of mFAST-SeqS is limited to chromosome arms, and no LINE-1 sequences are located in the FGFR1 amplified region, the amplification cannot be detected using mFAST-SeqS. However, high-level amplification can be detected with sWGS down to 1\% [23,24]. Therefore, we ran the same samples with sWGS irrespective of the mFAST-SeqS z-score (for detailed copy number profiles, see Supplementary data Figure S5). For copy number calling, we used our previously published plasma-Seq [15] approach, while the ichorCNA algorithm [13] was used to calculate the tumor fraction. A good correlation $\left(R^{2}=0.81\right)$ between $z$-score and ichorCNA estimates was observed (Figure 2), essentially driven by the high content tumor DNA cases.

Table 4. Amplification calling based on FISH, Oncoscan testing, and plasma-Seq data.

\begin{tabular}{cccccc}
\hline $\begin{array}{c}\text { Case No. } \\
\text { (Patient ID) }\end{array}$ & Biopsies-FISH & Oncoscan-Curie & $\begin{array}{c}\text { mFAST-SeqS } \\
\text { Z-Score }\end{array}$ & $\begin{array}{c}\text { ichorCNA } \\
\text { Tumor } \\
\text { Fraction (\%) }\end{array}$ & $\begin{array}{c}\text { FGFR1 Amplification } \\
\text { Calling Based on } \\
\text { Plasma-Seq Data }\end{array}$ \\
\hline 529014 & Amplified & Gain & 1.6 & 0.35 & - \\
529773 & Balanced & No Data & 10.6 & 38 & - \\
530077 & Balanced & No Data & 1.7 & 1.15 & - \\
531084 & Amplified & Gain & 4.5 & 4.6 & + \\
542827 & Amplified & No gain & 1.6 & 1.2 & - \\
544065 & Balanced & No Data & 31.5 & 47 & - \\
548696 & Balanced & No Data & 2.9 & 19 & - \\
557513 & Amplified & Gain & 1.7 & 3.9 & - \\
562315 & Amplified & Gain & 1.9 & 0.86 & - \\
568895 & Balanced & No Data & 1.1 & 0.6 & + \\
578098 & Balanced & No Data & 2 & 0.73 & - \\
587002 & Amplified & Gain & 3.8 & 9.5 & + \\
590243 & Balanced & No Data & 1.9 & 5.9 & + \\
594270 & Amplified & Gain & 2.4 & 5.5 & - \\
600575 & Amplified & Gain & 3.6 & 4.6 & + \\
612988 & Balanced & No Data & 1.8 & 0.7 & - \\
615064 & Amplified low & No Data & 19.7 & 35 & + \\
615972 & Balanced & No Data & 3.1 & 7.77 & - \\
623854 & Amplified & Gain & 11.1 & 16 & - \\
628696 & Balanced & No Data & 1.6 & 2.2 & \\
\hline
\end{tabular}

It is well established that SCNA analysis from cfDNA requires a minimal ctDNA AF. For the ichorCNA algorithm, a lower limit of 0.03 tumor fraction has been reported to detect the presence of tumors with high sensitivity (0.95) and specificity (0.91) [17]. From the 20 samples, for which we conducted plasma analyses, 12 had a ctDNA AF above 3\%, 7 of them had an FGFR1 amplification, whereas the other 5 cases had no copy number alteration of the FGFR1 region. In all of these 12 cases, the presence or absence of the FGFR1 amplification was correctly identified.

According to ichorCNA, the other three cases with FGFR1 amplifications had low ctDNA AFs of $0.35 \%$ (529014), $1.2 \%$ (542827), or $0.86 \%$ (562315). However, despite the low ctDNA AF of $0.86 \%$, the FGFR1 amplification was correctly identified in case 562315. Not surprisingly, the tissue evaluation of this case showed by far the highest number of signals, i.e., 25.1 (FISH) or 24 (Oncoscan) (Figure 3). This is a case in point that accurate resolution limits for amplifications are hard to establish as they 
critically depend on the level of amplification. In contrast, in case 529014, the ctDNA AF of $0.35 \%$ and about seven FGFR1 copies/nucleus (FISH and Oncoscan) were apparently insufficient for the detection of the amplification. In the third case 542827, the results between FISH and Oncoscan had already been inconsistent so that the presence of the amplification was questionable (Figure 3). When we correlated the $\log 2$ ratio of the $1 \mathrm{Mb}$ window harboring the FGFR1 gene, we observed a low correlation with the tumor fraction assessed with ichorCNA (iTF) for the amplified cases, and, as expected, no correlation for non-amplified cases of the respective region was balanced (or maybe only affected by a large gain) regardless of the tumor fraction (Supplementary data Figure S6). Additionally, we calculated the (hypothetical) absolute copy numbers of FGFR1 based on the log2ratio and tumor fraction estimation from ctDNA. Except for one outlier, these hypothetical copy numbers were highly correlated to Oncoscan copy number but not to FISH (Supplementary data Figure S7).

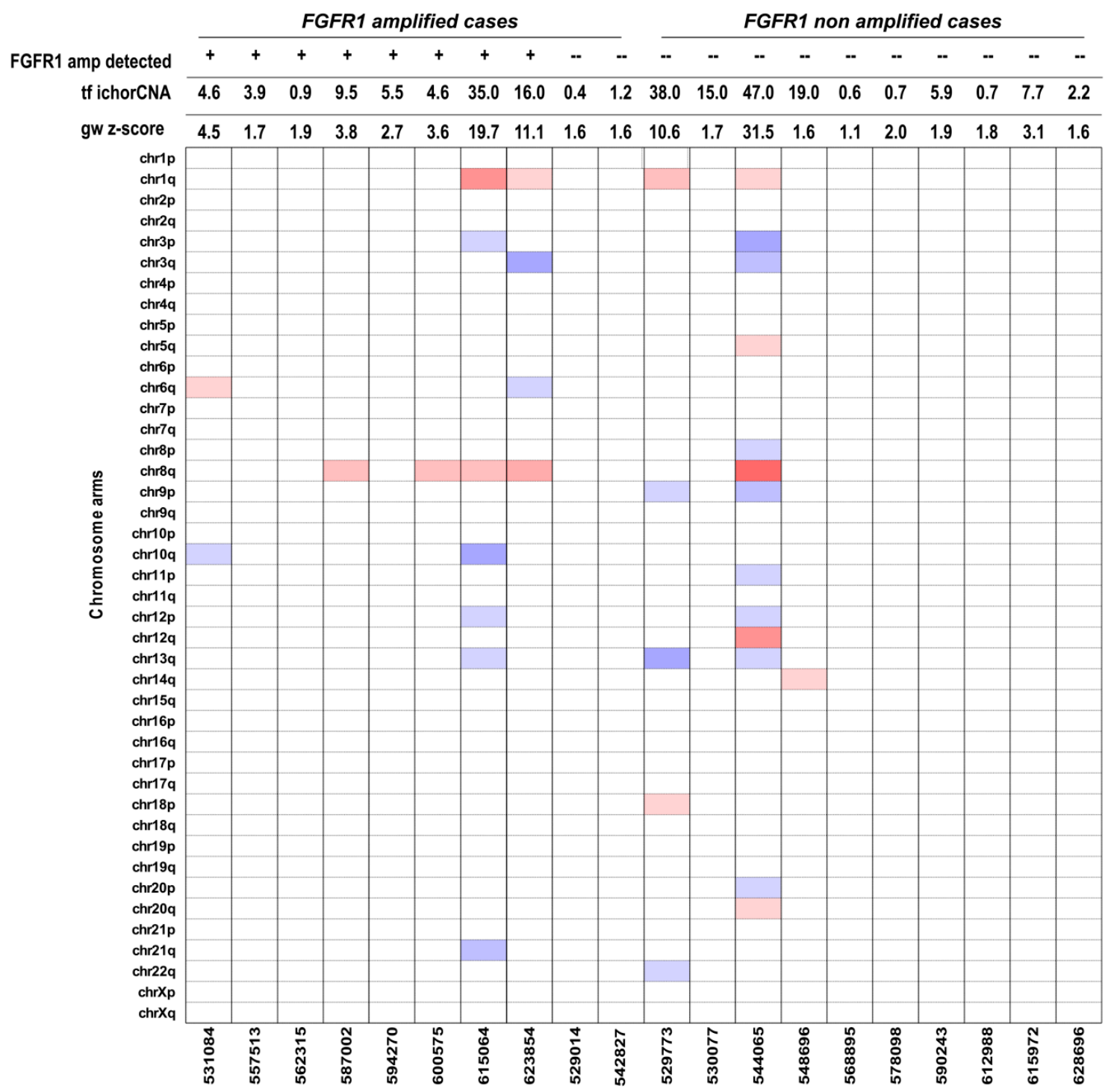

Figure 1. mFAST-SeqS (modified fast aneuploidy screening test-sequencing system) LINE-1 profiles for FGFR1 amplified $(n=10)$ and non-amplified cases $(n=10)$. Heat-map of selected plasma samples with different values of genome-wide z-scores. Blue bars indicate chromosome-specific Z-scores $<5$, and red bars indicate chromosome-specific z-scores $>5$. Amongst the 20 samples analyzed with mFAST-SeqS, the $\mathrm{z}$-score ranged from 1.1 to 31.5 with a median of $2.2 \mathrm{~m}$ with only 4 samples showing a Z-score $>5$. 


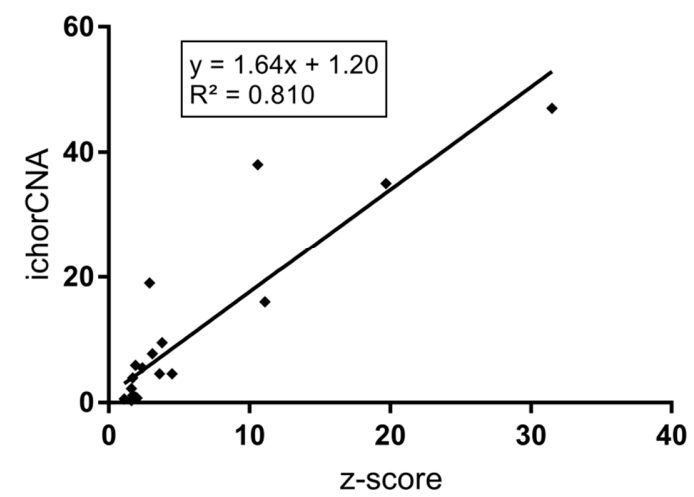

Figure 2. Correlation $\left(\mathrm{R}^{2}\right.$ of 0.81$)$ between mFAST-SeqS z-score and ichorCNA.

Since the detection of FGFR1 amplification in plasma critically depends on both the absolute copy number of the gene (amplification amplitude) and then tumor fractions, a generalized threshold for the detection could not be established.

\section{3. cfDNA Tumor Fraction Could Predict Survival}

Previous reports using targeted methodologies (dPCR, ddPCR, and targeted-NGS) [25] and a recent study with mFAST-SeqS [26] have demonstrated that higher tumor levels in plasma are indicative of a worse prognosis in breast cancer. In this report, we wished to demonstrate the suitability of evaluating tumor fraction by ichorCNA using ULP-WGS in a metastatic breast cancer cohort. First, we used the median genome-wide z-score for stratification. Indeed, we found that $\mathrm{z}$-scores above the median of 2.2 were associated with significantly decreased overall survival (OS) ( $p$-value for the log-rank test; $p=0.009$ ), yet with slightly overlapping CI (Figure 4a). Likewise, splitting patients, based on the median tumor fraction established with ichorCNA (ichorCNA median 4.6), led to a similar separation of the Kaplan-Meier curves ( $p$-value for the log-rank test; $p<0.001$ ) (Figure $4 \mathrm{~b}$ ). On the contrary, the total cfDNA quantity estimated by Qubit (based on median cut-off) was not found relevant to discriminate OS (Figure 4c).

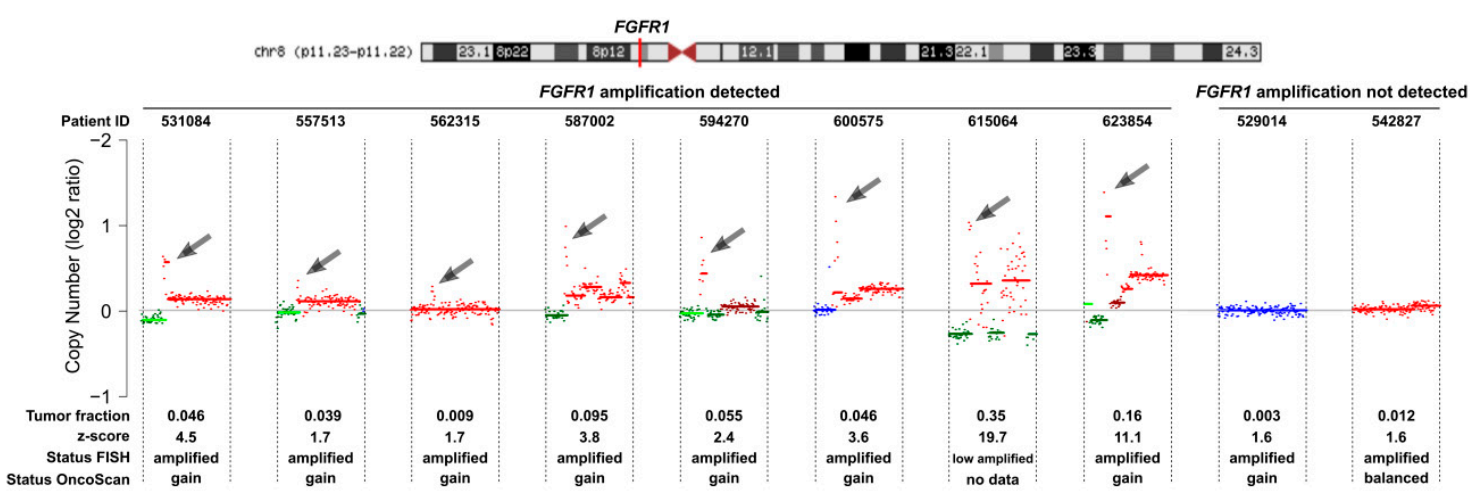

Figure 3. Copy number status of chromosome 8 of cell-free DNA (cfDNA) samples established with shallow whole-genome sequencing (sWGS). The log-2 ratios of chromosomes 8 of plasma samples from 10 metastatic breast cancer patients with FGFR1 amplification in their tumors are plotted. In 8 of those, the FGFR1 amplification could also be detected in plasma (right panel), whereas in 2 cases, the amplification could not be detected most likely due to a low tumor content. The gain of chromosomal regions is indicated in red, losses are shown in green, and balanced regions are shown in blue. Tumor fraction estimated from ichorCNA, z-score, FISH, and Oncoscan status is indicated. 

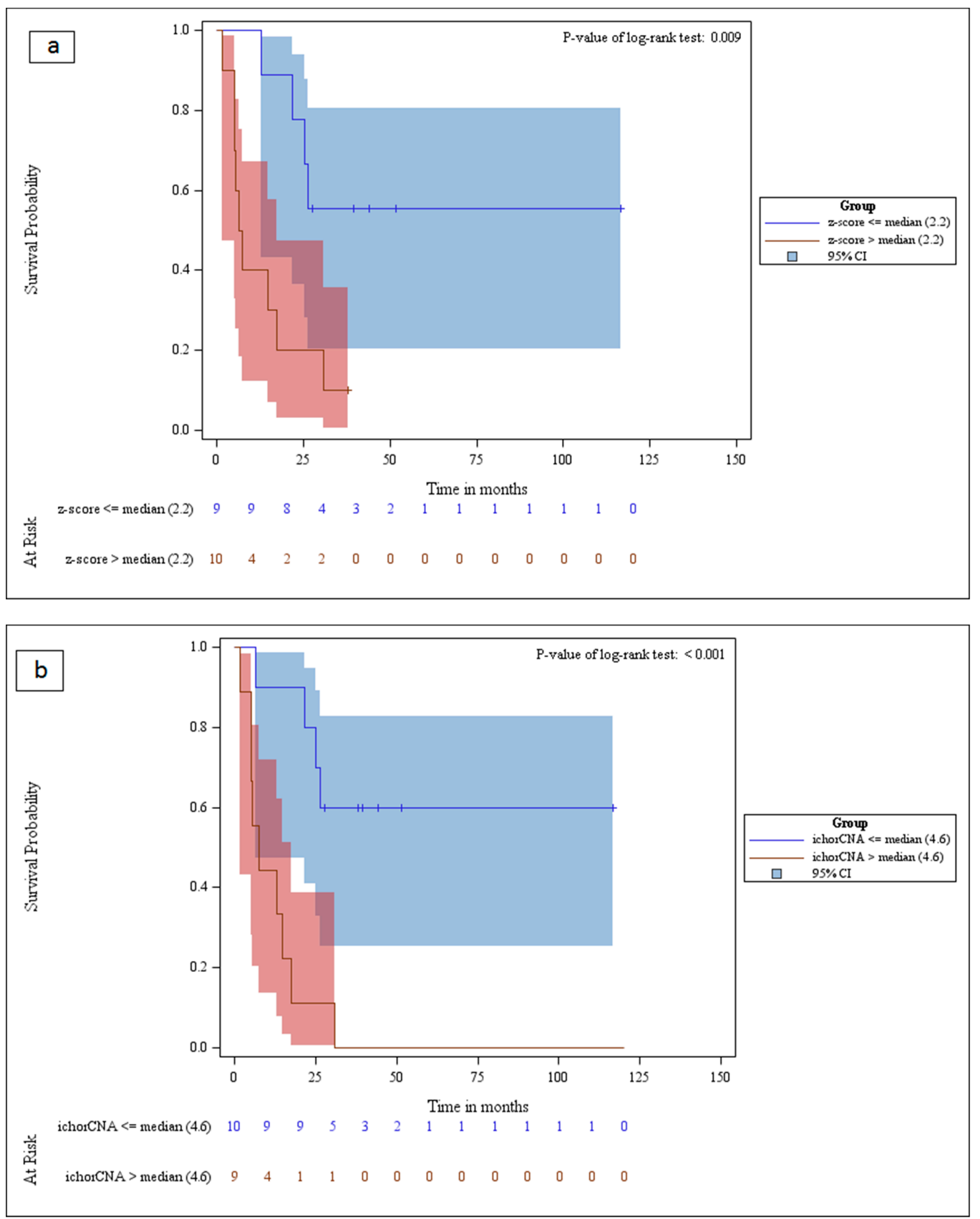

Figure 4. Cont. 


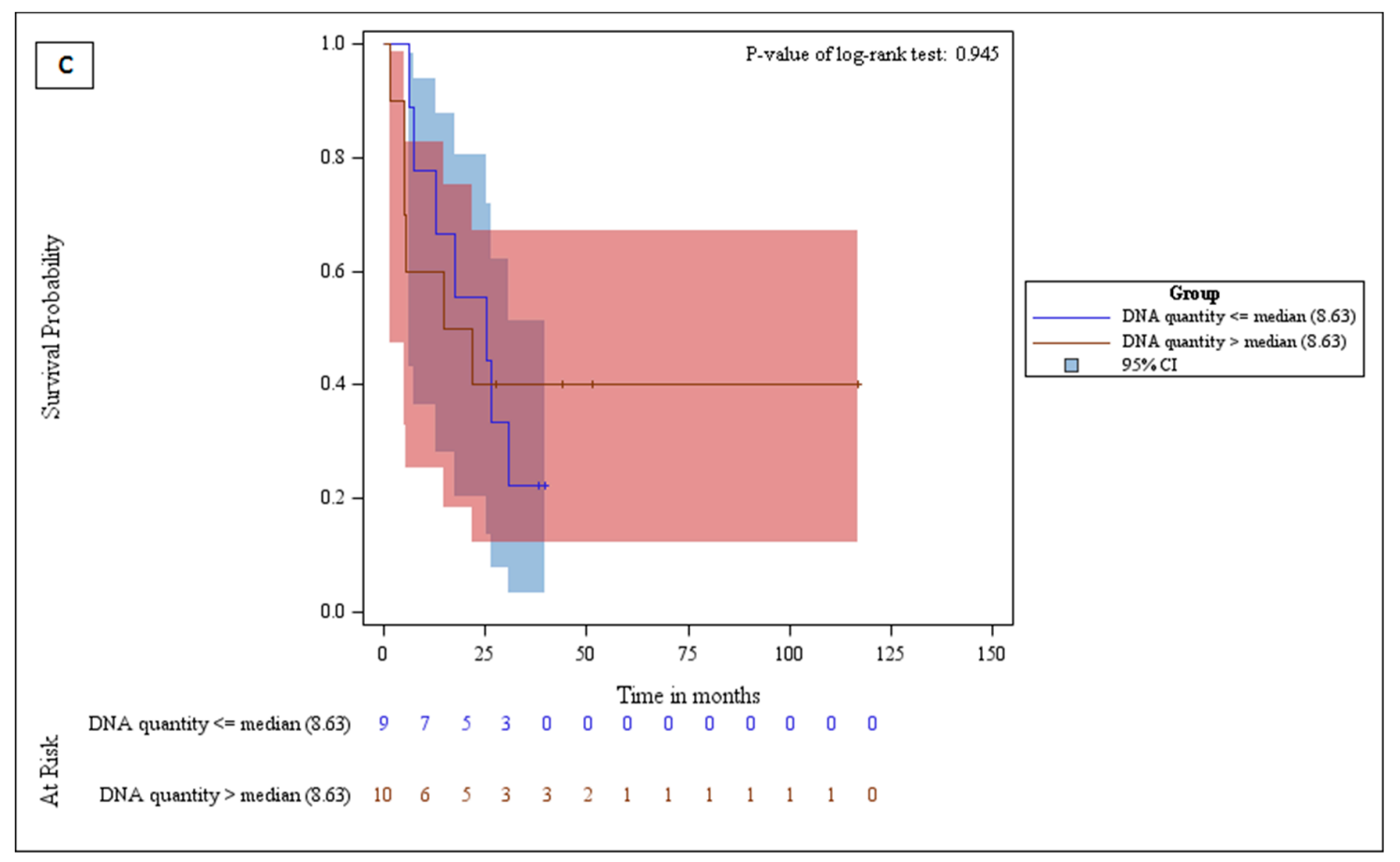

Figure 4. Kaplan-Meier analysis curves for the overall survival of metastatic breast cancer patients. (a) Overall survival of patients stratified by the median mFAST-SeqS z-score, (b) Overall survival of patients stratified by the median tumor fraction calculated with ichorCNA cut-off, (c) Overall survival of patients stratified by the median cfDNA quantity established by Qubit.

\section{Discussion}

Despite the clear rationale for targeting FGFR1 in BC and multiple therapeutic strategies based on both selective and non-selective FGFR1 inhibitors, none have, to date, achieved an objective response. The moderate signs of efficacy could be the cumulative effect of ineffective compounds, inadequate patient selection, or the lack of oncogenic potential of FGFR1.

In this study, we successfully demonstrated the feasibility of sWGS (plasma-Seq) for detecting genome-wide structural genomic anomalies, including FGFR1 gene amplification, as well as estimating tumor content in the plasma of $\mathrm{mBC}$ patients. Despite the small sample size, good concordance was observed between FGFR1 amplification in plasma assessed from sWGS data and the tissue-based analysis (FISH and Onscoscan ${ }^{\mathrm{TM}}$ ). Therefore, the method has the potential to directly screen and detect FGFR1 amplification in a non-invasive and specific manner in plasma samples in patients with advanced metastatic disease, demonstrating significant tumor burden.

Amplification calling in plasma, compared to mutation detection, is much more challenging, where the ratio between the ctDNA and normal cfDNA determines the capacity of defining amplification status. Indeed, several targeted NGS methods based on amplicon-based multiplexed PCR and hybrid-capture have been described for detecting specific SCNAs [27], but high coverage and in-depth sequencing requirements, as well as lack of cost-effectiveness, have somewhat limited their widespread clinical implementation. In addition, cost-effective methods for detecting SCNAs in cfDNA based on ddPCR have so far only been described for ERBB2 (HER2) [28] and FGFR2 [29]. Although sWGS can specifically detect both focal and gross copy number changes, its major limitation is the lack of sensitivity to detect low copy number gain/loss and smaller genomic changes due to the low tumor DNA component in early-stage cancer. In addition, another limitation in using sWGS in estimating plasma copy numbers is that only relative copy number changes are measured, which neither allows establishing the ploidy level nor the exact prediction of the absolute copy number due to the dilution effects with DNA from normal cells [15]. Despite these shortcomings-and although the evaluation of cost-effectiveness and duration were not within the scope of the study—sWGS (and mFAST-SeqS) still 
represented a pan-cancer, low-cost, and rapid screening approach to specifically detect global genomic structural anomalies in cfDNA to enable the identification of actionable therapy and also monitoring in advanced cancer patients. The turnaround time of these methods is less than two days at costs of less than 100EUR. Further validation in a larger cohort will be required to reinforce the potential of this method for screening the FGFR1 amplification.

In addition, and despite the small cohort size, both untargeted sequencing methods-mFAST-SeqS and sWGS - demonstrated similar results in predicting survival based on tumor content in metastatic breast cancer (mBC) patients. Although the estimation of tumor fraction in plasma with each method (mFAST-SeqS and ichorCNA) demonstrated high correlation, ichorCNA might be a more accurate representation of the tumor fraction and, therefore, enable a better stratification $(p<0.001$ versus $p=0.009$ ). mFAST-SeqS is based on LINE-1 read counts, and the z-score depends on the overall amount of copy number alterations, as well as the amplitude of copy number alterations, while ichorCNA interrogates the entire genome. The specificity of these methods is reinforced by the fact that measuring global cfDNA levels in the plasma of the same patients is not predictive of survival. Both methods have been previously proven to be of prognostic value in breast cancer $[17,26]$. The mFAST-SeqS-based assessment has also been used in the prostate [24,30] and lung cancer [31]. Highly concordant copy number profiles of mFAST-SeqS and plasma-Seq were observed in patients' plasma samples with z-score $>3$. However, in contrast to sWGS, mFAST-SeqS did not allow to call for FGFR1 amplification, as there was no LINE-1 sequence near the FGFR1 gene.

Considering the entire cohort of 100 patients, we confirmed previous reports that FGFR1 amplification based on tissue analyses is associated with significantly worse survival $[5,6,9,32,33]$. Unfortunately, due to the limited number of patients with available plasma, we were unable to perform a similar analysis in plasma with sufficient statistical power.

\section{Materials and Methods}

\subsection{Clinicopathologic Data and Sample Collection}

Metastatic breast tumors and blood samples were selected from 100 female patients at the Institute Curie, Paris, based on the availability of both tissue and plasma samples (Table 1). Blood sampling was performed at diagnosis of metastasis or before the beginning of a new line of treatment for metastatic disease. In accordance with the French regulation, written consent was obtained from all patients/guardians on specimens. This research has been approved by the institutional review board of the Institut Curie on 11 June 2015. The patients were treated for stage IV disease at the Institute Curie, Paris, from 4/2006 to 4/2018, with a median follow-up of 6 years and a median overall survival of 44 months. All patients received systemic treatment (endocrine and/or chemotherapy) according to tumor characteristics and standard of care at the time of inclusion. The median age of patients in the cohort was 60y (range 28-83). The details of the clinical and histopathological data care are summarized in Table 1. Tissue samples were fixed in formalin, embedded in paraffin (FFPE), and stored under standard conditions. Blood was drawn into standard EDTA tubes. Plasma was extracted by centrifugation at $2000 \mathrm{rpm}$ for $10 \mathrm{~min}$, followed by careful aliquotting and freezing at $-80{ }^{\circ} \mathrm{C}$ within 1-24 $\mathrm{h}$ after collection.

\subsection{Fluorescence In Situ Hybridization (FISH)}

FISH was performed on $5 \mu \mathrm{m}$ tumor sections. We used a commercially available and standardized probe for the detection of FGFR1 (Zytovision ${ }^{\mathrm{TM}}$ ), and hybridization was performed according to the manufacturer's instructions. FGFR1 was assessed at a central facility (Zytovision ${ }^{\mathrm{TM}}$ ) on available archived metastatic biopsy. Where possible, we scored 50 cells per sample for hybridization patterns. FGFR1 amplification was defined, based on published criteria [22]; notably, the FGFR1/CEN8 ratio was $\geq 2.0$, or the average number of FGFR1 signals per tumor cell nucleus was $\geq 6$. Additional evaluation 
criteria (FGFR1 low amplification) were considered if the percent of tumor cells containing $>5$ gene signals was $>50 \%$ and/or $\%$ of tumor cells containing $>15$ gene signals or large clusters was $>10 \%$.

\subsection{Genomic Oncoscan Profile}

DNA was isolated from FFPE tumor samples using the GeneRead DNA FFPE Extraction Kit (Qiagen, Hilden, Germany), following the manufacturer's guidelines. The genome-wide copy number profile, including regions with loss of heterozygosity $(\mathrm{LOH})$, was established using Oncoscan ${ }^{\mathrm{TM}}$ arrays, according to the supplier's recommendation (Affymetrix-Thermofisher Scientific, Inc., Santa Clara, CA, USA, ONCOSCAN CNV FFPE ASSAY, ref. 902293) and as described previously [34,35]. Data analysis was performed using Chromosome Analysis Suite Software from Affymetrix (CHAS), version 3.0.0.42, with annotations na33.1 (hg19). QC metrics were generated and gave information about the data quality and the level of chromosomal aberrations. Comprehensive Pipeline for Analyzing and Visualizing Array-Based CGH Data R Package was used for whole-genome representation [36] (rCGH: version 1.14.0).

\subsection{Cell-Free DNA Extraction and Quantification}

cfDNA was extracted from 1 to $4 \mathrm{~mL}$ of plasma using Maxwell RSC ccfDNA Plasma Kit (RSC; Promega, Leiden, the Netherlands), according to the manufacturers' recommendation. The RSC kit is a magnetic beads-based method, fully automated, including a lysis step before DNA binding to magnetic beads. cfDNA concentration was measured by Qubit Fluorometric Assay (Thermo Fisher Scientific, Aalst, Belgium) with dsDNA HS (High Sensitivity) Assay Kit. The total cfDNA quantity per $\mathrm{mL}$ of plasma was calculated.

\section{5. mFAST-SeqS}

To stratify patients based on their tumor levels, mFAST-SeqS was used, as previously described [30,37]. Briefly, LINE-1 (L1) amplicon libraries were prepared from 5-10 $\mu \mathrm{L}$ of plasma DNA corresponding to $0.1-5$ ng total DNA using Phusion Hot Start II Polymerase in 5 PCR cycles with target-specific L1 primers. The $10 \mu \mathrm{L}$ of the purified (AMPure Beads, Beckman Coulter, Brea, CA, USA) PCR products were used for the addition of Illumina-specific sequencing adaptors and indices. L1 amplicon libraries were sequenced on an Illumina MiSeq, generating $150 \mathrm{bp}$ single reads aiming for at least 100,000 reads per sample. Sequence reads were aligned to the hg19 genome, and reads with a mapping quality $>15$ were counted per chromosome arm with an in-house script. Normalized read counts were compared to a control population $(n=35)$ using z-score statistics by subtracting the mean and dividing by the SD to assess over- and under-representation of LINE-1-sequences. The short arms of acrocentric chromosomes were omitted from the analysis. Finally, all chromosome arm-specific z-scores were squared and summed up, resulting in a genome-wide z-score, which acts as a surrogate for tumor fraction [30].

\subsection{Shallow Whole-Genome Sequencing sWGS (PLASMA-SEQ)}

For genome-wide copy number profiling, sWGS (plasma-Seq) was performed, as previously described [14]. Briefly, shotgun libraries were prepared from 5-10 ng plasma DNA using the TruSeq DNA Nano Sample Preparation Kit (Illumina, Inc., San Diego, CA, USA). Due to the fragmentation of plasma DNA and the low input amounts, we omitted the fragmentation step and increased the number of PCR cycles to 20. Libraries were quality checked on an Agilent Bioanalyzer (Agilent, Santa Clara, CA, USA) and quantified using qPCR. Finally, libraries were sequenced on an Illumina MiSeq or NextSeq with an average of 6.43 mio. reads (range 4.7-8.5 mio.) per sample. Large copy number changes, as well as focal events, were established using our optimized in-house plasma-Seq script [14,24]. FGFR1 copy number status was evaluated based on the previously published criteria for focal amplification calling. Among other genes, such as AR ERBB2, MET, and MYC, FGFR1 amplification was already validated using quantitative real-time [22,38]. Additionally, data were analyzed with the previously published 
ichorCNA algorithm to calculate tumor fractions (iTF) from sWGS data [17]. The hypothetical absolute copy number $(\mathrm{ACN})$ of the $1 \mathrm{Mb}$ window harboring the FGFR1 gene was calculated by formula (2): $\mathrm{ACN}=(2 *(2 \wedge \log 2$ Ratio $+\mathrm{iTF}-1)) / \mathrm{iTF}[39]$.

\subsection{Statistical Analysis}

Descriptive statistics were used in order to analyses the relationship between variables. Kaplan-Meier analysis was used to estimate overall survival. To stratify patients, the median values of the genome-wide $\mathrm{z}$-score assessed with mFAST-SeqS and the tumor fraction calculated from ichorCNA were used. Survival was defined as the time from the date of measurement for ctDNA data to the date of death, the date of last news, or to 26 January 2018 (right censoring). The Kaplan-Meier curves and their $95 \%$ confidence intervals, as well as the survival medians (in months), were presented by the group. All statistical analyses were performed using SAS ${ }^{\circledR} /$ PC Software version 9.2.

For all the correlations presented, Pearson correlation coefficients were performed with GraphPad Prism.

\section{Conclusions}

In summary, we demonstrated the feasibility of using sWGS as an assay to detect clinically informative SCNA, such as FGFR1 amplification, in plasma of mBC patients. In the cases where access to fresh biopsies is not possible or that only archived samples are available, sWGS could be used for screening or re-evaluating the amplification status of the patients. In addition, both mFAST-SeqS and sWGS confirmed their potential in a broad-based application for the estimation of tumor fraction and, thus, tumor monitoring in advanced cancer patients.

Supplementary Materials: The following are available online at http:/www.mdpi.com/2072-6694/12/6/1481/ s1, Figure S1: Kaplan-Meier log-rank $p=0.01$ overall survival according to FGFR1 amplification status, Figure S2: Kaplan-Meier log-rank $p=0.01$ overall survival according to histological subtype (log-rank), Figure S3: Kaplan-Meier $\log$-rank $p=0.035$ overall survival according to the disease-free interval in months (log-rank), Figure S4: Pearson correlation analysis $\left(\mathrm{R}^{2}\right.$ of 0.331$)$ between FGFR1 copy number determined by Oncoscan and FISH results (Average number of FGFR1 signals per tumor cell nucleus), Figure S5: Detailed copy number profiles for each patient sample obtained by sWGS, Table S1: FGFR1 amplified cases based on FISH testing of a metastatic breast cancer cohort $(n=100)$. * FGFR1 amplification was based on the criteria of Schildhaus et al., 2012. Notably: the FGFR1/CEN8 ratio was $\geq 2.0$, or the average number of FGFR1 signals per tumor cell nucleus was $\geq 6$. Additional evaluation criteria (FGFR1 low amplification) were considered if the $\%$ of tumor cells containing $>5$ gene signals was $>50 \%$ and/or $\%$ of tumor cells containing $>15$ gene signals or large clusters was $>10 \%$. Figure S6: Correlation of tumour fractions FGFR1 log2ratios in plasma. Shown is a linear regression of the ichorCNA-derived tumour fraction (iTF) and respective log2ratio of a $1 \mathrm{Mb}$ genomic region harbouring the FGFR1 gene. Left plot, plasma DNA data from FGFR1 amplified cases. Right plot, plasma DNA data from FGFR1 non-amplified cases. Figure S7: Correlation of copy numbers from three orthogonal approaches. Shown is a linear regressions of the copy number (CN) established from FISH, ONCOSCAN and sWGS. Hypothetical absolute copy numbers $(\mathrm{ACN})$ were calculated form the ichorCNA-derived tumour fraction (iTF) and respective log2ratio for FGFR1. Upper plot, Correlation of FISH and ACN from ichorCNA. Lower left plot, Correlation of ONCOSCAN and ACN from ichorCNA. Lower right plot, Correlation of ONCOSCAN and ACN from ichorCNA without one outlier.

Author Contributions: Conceptualization: N.G.-S., B.P.L., C.B., L.X., E.H., M.R.S., and J.-Y.P.; Methodology: N.G.-S., B.P.L., C.B., L.X., E.H., M.R.S., J.-Y.P., and J.B.; Software: C.B., E.H., J.B., L.X., and H.S.; Validation: N.G.-S., B.P.L., C.B., L.X., E.H., M.R.S., J.-Y.P., Formal Analysis: N.G.-S., B.P.L., C.B., L.X., E.H., M.R.S., J.-Y.P., H.S., J.B., C.P., Investigation: N.G.-S., B.P.L., C.B., L.X., E.H., M.R.S., J.-Y.P., H.S., and J.B.; Data Curation: N.G.-S., C.B., L.X., E.H., J.-Y.P., H.S., and J.B.; Supervision: N.G.-S., B.P.L., E.H., and J.-Y.P.; Writing-Original Draft Preparation: N.G.-S, B.P.L., and C.B.; Writing-Review and Editing: N.G.-S, B.P.L., C.B., L.X., and E.H.; Funding Acquisition: B.P.L.; All authors have read and agreed to the published version of the manuscript.

Funding: This research was funded by Servier Research collaboration with the Curie Institute and through the Cancer-ID IMI-Program with the University of Graz.

Acknowledgments: We thank Dominique Rostagni and Bettina da Silva for their support for the management of clinical samples and references.

Conflicts of Interest: Hélène Salaun, Chantal Bourrier, Nolwen Guigal-Stephan, Brian Lockhart, and Laura Xuereb declare no conflict of interest. C. Proudhon and J.Y. Pierga have an unrelated sponsored research agreement with Servier within CANCER-ID, a project funded by the Innovative Medicines Joint Undertaking (IMI JU). 
Ellen Heitzer and Michael R. Speicher have an unrelated sponsored research agreement with Servier within CANCER-ID, a project funded by the Innovative Medicines Joint Undertaking (IMI JU); the salary of Jelena Belic was paid through this arrangement. E.H. receives funding from Freenome, South San Francisco, CA, and PreAnalytiX, Hombrechtikon, Switzerland.

\section{References}

1. Perez-Garcia, J.; Muñoz-Couselo, E.; Soberino, J.; Racca, F.; Cortes, J. Targeting FGFR pathway in breast cancer. Breast 2018, 37, 126-133. [CrossRef] [PubMed]

2. Elbauomy Elsheikh, S.; Green, A.R.; Lambros, M.B.K.; Turner, N.C.; Grainge, M.J.; Powe, D.; Ellis, I.O.; Reis-Filho, J.S. FGFR1 amplification in breast carcinomas: A chromogenic in situ hybridisation analysis. Breast Cancer Res. 2007, 9, R23. [CrossRef] [PubMed]

3. Gelsi-Boyer, V.; Orsetti, B.; Cervera, N.; Finetti, P.; Sircoulomb, F.; Rougé, C.; Lasorsa, L.; Letessier, A.; Ginestier, C.; Monville, F.; et al. Comprehensive profiling of 8p11-12 amplification in breast cancer. Mol. Cancer Res. 2005, 3, 655-667. [CrossRef] [PubMed]

4. Chang, J.; Liu, X.; Wang, S.; Zhang, Z.; Wu, Z.; Zhang, X.; Li, J. Prognostic value of FGFR gene amplification in patients with different types of cancer: A systematic review and meta-analysis. PLoS ONE 2014, 9, e105524. [CrossRef] [PubMed]

5. Turner, N.; Pearson, A.; Sharpe, R.; Lambros, M.; Geyer, F.; Lopez-Garcia, M.A.; Natrajan, R.; Marchio, C.; Iorns, E.; Mackay, A.; et al. FGFR1 amplification drives endocrine therapy resistance and is a therapeutic target in breast cancer. Cancer Res. 2010, 70, 2085-2094. [CrossRef] [PubMed]

6. Carene, D.; Tran-Dien, A.; Lemonnier, J.; Dalenc, F.; Levy, C.; Pierga, J.-Y.; Jacot, W.; Canon, J.-L.; Richon, C.; Lacroix, L.; et al. Association between FGFR1 copy numbers, MAP3K1 mutations, and survival in axillary node-positive, hormone receptor-positive, and HER2-negative early breast cancer in the PACS04 and METABRIC studies. Breast Cancer Res. Treat. 2020, 179, 387-401. [CrossRef] [PubMed]

7. Lee, H.J.; Seo, A.N.; Park, S.Y.; Kim, J.Y.; Park, J.Y.; Yu, J.H.; Ahn, J.-H.; Gong, G. Low prognostic implication of fibroblast growth factor family activation in triple-negative breast cancer subsets. Ann. Surg. Oncol. 2014, 21, 1561-1568. [CrossRef]

8. Shi, Y.-J.; Tsang, J.Y.S.; Ni, Y.-B.; Chan, S.-K.; Chan, K.-F.; Tse, G.M. FGFR1 is an adverse outcome indicator for luminal A breast cancers. Oncotarget 2016, 7, 5063-5073. [CrossRef]

9. Jang, M.; Kim, E.; Choi, Y.; Lee, H.; Kim, Y.; Kim, J.; Kang, E.; Kim, S.-W.; Kim, I.; Park, S. FGFR1 is amplified during the progression of in situ to invasive breast carcinoma. Breast Cancer Res. 2012, 14, R115. [CrossRef]

10. Rueda, O.M.; Sammut, S.-J.; Seoane, J.A.; Chin, S.-F.; Caswell-Jin, J.L.; Callari, M.; Batra, R.; Pereira, B.; Bruna, A.; Ali, H.R.; et al. Dynamics of breast-cancer relapse reveal late-recurring ER-positive genomic subgroups. Nature 2019, 567, 399-404. [CrossRef]

11. Drago, J.Z.; Formisano, L.; Juric, D.; Niemierko, A.; Servetto, A.; Wander, S.A.; Spring, L.M.; Vidula, N.; Younger, J.; Peppercorn, J.; et al. FGFR1 amplification mediates endocrine resistance but retains TORC sensitivity in metastatic hormone receptor-positive (HR+). Breast Cancer. Clin. Cancer Res. 2019, 25, 6443-6451. [CrossRef] [PubMed]

12. Yates, L.R.; Knappskog, S.; Wedge, D.; Farmery, J.H.R.; Gonzalez, S.; Martincorena, I.; Alexandrov, L.B.; Van Loo, P.; Haugland, H.K.; Lilleng, P.K.; et al. Genomic evolution of breast cancer metastasis and relapse. Cancer Cell. 2017, 32, 169-184. [CrossRef] [PubMed]

13. Yoshida, A.; Hayashi, N.; Suzuki, K.; Takimoto, M.; Nakamura, S.; Yamauchi, H. Change in HER2 status after neoadjuvant chemotherapy and the prognostic impact in patients with primary breast cancer. J. Surg. Oncol. 2017, 116, 1021-1028. [CrossRef] [PubMed]

14. Heitzer, E. Circulating tumor DNA for modern cancer management. Clin. Chem. 2019, 66, 143-145. [CrossRef]

15. Heitzer, E.; Ulz, P.; Belic, J.; Gutschi, S.; Quehenberger, F.; Fischereder, K.; Benezeder, T.; Auer, M.; Pischler, C.; Mannweiler, S.; et al. Tumor-associated copy number changes in the circulation of patients with prostate cancer identified through whole-genome sequencing. Genome Med. 2013, 5, 30. [CrossRef]

16. Mohan, S.; Heitzer, E.; Ulz, P.; Lafer, I.; Lax, S.; Auer, M.; Pichler, M.; Gerger, A.; Eisner, F.; Hoefler, G.; et al. Changes in colorectal carcinoma genomes under anti-EGFR therapy identified by whole-genome plasma DNA sequencing. PLoS Genet. 2014, 10, e1004271. [CrossRef] 
17. Adalsteinsson, V.A.; Ha, G.; Freeman, S.S.; Choudhury, A.D.; Stover, D.G.; Parsons, H.A.; Gydush, G.; Reed, S.C.; Rotem, D.; Rhoades, J.; et al. Scalable whole-exome sequencing of cell-free DNA reveals high concordance with metastatic tumors. Nat. Commun. 2017, 8, 1324. [CrossRef]

18. Hovelson, D.H.; Liu, C.-J.; Wang, Y.; Kang, Q.; Henderson, J.; Gursky, A.; Brockman, S.; Ramnath, N.; Krauss, J.C.; Talpaz, M.; et al. Rapid, ultra low coverage copy number profiling of cell-free DNA as a precision oncology screening strategy. Oncotarget 2017, 8, 89848-89866. [CrossRef]

19. Stover, D.G.; Parsons, H.A.; Ha, G.; Freeman, S.S.; Barry, W.T.; Guo, H.; Choudhury, A.D.; Gydush, G.; Reed, S.C.; Rhoades, J.; et al. Association of cell-free DNA tumor fraction and somatic copy number alterations with survival in metastatic triple-negative breast cancer. J. Clin. Oncol. 2018, 36, 543-553. [CrossRef]

20. Manier, S.; Park, J.; Capelletti, M.; Bustoros, M.; Freeman, S.S.; Ha, G.; Rhoades, J.; Liu, C.J.; Huynh, D.; Reed, S.C.; et al. Whole-exome sequencing of cell-free DNA and circulating tumor cells in multiple myeloma. Nat. Commun. 2018, 9, 1691. [CrossRef]

21. Foulkes, W.D.; Smith, I.E.; Reis-Filho, J.S. Triple-negative breast cancer. N. Engl. J. Med. 2010, 363, $1938-1948$. [CrossRef] [PubMed]

22. Schildhaus, H.-U.; Heukamp, L.C.; Merkelbach-Bruse, S.; Riesner, K.; Schmitz, K.; Binot, E.; Paggen, E.; Albus, K.; Schulte, W.; Ko, Y.-D.; et al. Definition of a fluorescence in-situ hybridization score identifies highand low-level FGFR1 amplification types in squamous cell lung cancer. Mod. Pathol. 2012, 25, 1473-1480. [CrossRef] [PubMed]

23. Belic, J.; Koch, M.; Ulz, P.; Auer, M.; Gerhalter, T.; Mohan, S.; Fischereder, K.; Petru, E.; Bauernhofer, T.; Geigl, J.B.; et al. Rapid identification of plasma DNA samples with increased ctDNA levels by a modified FAST-SeqS approach. Clin. Chem. 2015, 61, 838-849. [CrossRef] [PubMed]

24. Ulz, P.; Belic, J.; Graf, R.; Auer, M.; Lafer, I.; Fischereder, K.; Webersinke, G.; Pummer, K.; Augustin, H.; Pichler, M.; et al. Whole-genome plasma sequencing reveals focal amplifications as a driving force in metastatic prostate cancer. Nat. Commun. 2016, 7, 12008. [CrossRef]

25. Tan, G.; Chu, C.; Gui, X.; Li, J.; Chen, Q. The prognostic value of circulating cell-free DNA in breast cancer: A meta-analysis. Medicine (Baltimore) 2018, 97, e0197. [CrossRef]

26. Suppan, C.; Brcic, I.; Tiran, V.; Mueller, H.D.; Posch, F.; Auer, M.; Ercan, E.; Ulz, P.; Cote, R.J.; Datar, R.H.; et al. Untargeted assessment of tumor fractions in plasma for monitoring and prognostication from metastatic breast cancer patients undergoing systemic treatment. Cancers 2019, 11, 1171. [CrossRef]

27. Elazezy, M.; Joosse, S.A. Techniques of using circulating tumor DNA as a liquid biopsy component in cancer management. Comput. Struct. Biotechnol. J. 2018, 16, 370-378. [CrossRef]

28. Gevensleben, H.; Garcia-Murillas, I.; Graeser, M.K.; Schiavon, G.; Osin, P.; Parton, M.; Smith, I.E.; Ashworth, A.; Turner, N.C. Noninvasive detection of HER2 amplification with plasma DNA digital PCR. Clin. Cancer Res. 2013, 19, 3276-3284. [CrossRef]

29. Pearson, A.; Smyth, E.; Babina, I.S.; Herrera-Abreu, M.T.; Tarazona, N.; Peckitt, C.; Kilgour, E.; Smith, N.R.; Geh, C.; Rooney, C.; et al. High-level clonal FGFR amplification and response to FGFR inhibition in a translational clinical trial. Cancer Discov. 2016, 6, 838-851. [CrossRef]

30. Belic, J.; Graf, R.; Bauernhofer, T.; Cherkas, Y.; Ulz, P.; Waldispuehl-Geigl, J.; Perakis, S.; Gormley, M.; Patel, J.; $\mathrm{Li}, \mathrm{W}$; et al. Genomic alterations in plasma DNA from patients with metastasized prostate cancer receiving abiraterone or enzalutamide. Int. J. Cancer 2018, 143, 1236-1248. [CrossRef]

31. de Wit, S.; Rossi, E.; Weber, S.; Tamminga, M.; Manicone, M.; Swennenhuis, J.F.; Groothuis-Oudshoorn, C.G.M.; Vidotto, R.; Facchinetti, A.; Zeune, L.L.; et al. Single tube liquid biopsy for advanced non-small cell lung cancer. Int. J. Cancer 2019, 144, 3127-3137. [CrossRef] [PubMed]

32. Gru, A.A.; Allred, D.C. FGFR1 amplification and the progression of non-invasive to invasive breast cancer. Breast Cancer Res. 2012, 14, 116. [CrossRef] [PubMed]

33. Formisano, L.; Lu, Y.; Servetto, A.; Hanker, A.B.; Jansen, V.M.; Bauer, J.A.; Sudhan, D.R.; Guerrero-Zotano, A.L.; Croessmann, S.; Guo, Y.; et al. Aberrant FGFR signaling mediates resistance to CDK4/6 inhibitors in ER+ breast cancer. Nat. Commun. 2019, 10, 1373. [CrossRef] [PubMed]

34. Piqueret-Stephan, L.; Marcaillou, C.; Reyes, C.; Honoré, A.; Letexier, M.; Gentien, D.; Droin, N.; Lacroix, L.; Scoazec, J.-Y.; Vielh, P. Massively parallel DNA sequencing from routinely processed cytological smears. Cancer Cytopathol. 2016, 124, 241-253. [CrossRef] [PubMed] 
35. Foster, J.M.; Oumie, A.; Togneri, F.S.; Vasques, F.R.; Hau, D.; Taylor, M.; Tinkler-Hundal, E.; Southward, K.; Medlow, P.; McGreeghan-Crosby, K.; et al. Cross-laboratory validation of the OncoScan®FFPE Assay, a multiplex tool for whole genome tumour profiling. BMC Med. Genomics 2015, 8, 5. [CrossRef]

36. Commo, F.; Guinney, J.; Ferté, C.; Bot, B.; Lefebvre, C.; Soria, J.-C.; André, F. rCGH: A comprehensive array-based genomic profile platform for precision medicine. Bioinformatics 2016, 32, 1402-1404. [CrossRef]

37. Kinde, I.; Papadopoulos, N.; Kinzler, K.W.; Vogelstein, B. FAST-SeqS: A simple and efficient method for the detection of aneuploidy by massively parallel sequencing. PLOS ONE 2012, 7, e41162. [CrossRef]

38. Zhou, Q.; Perakis, S.O.; Ulz, P.; Mohan, S.; Riedl, J.M.; Talakic, E.; Lax, S.; Tötsch, M.; Hoefler, G.; Bauernhofer, T.; et al. Cell-free DNA analysis reveals POLR1D-mediated resistance to bevacizumab in colorectal cancer. Genome Med. 2020, 12, 20. [CrossRef]

39. Peng, H.; Lu, L.; Zhou, Z. CNV Detection from Circulating Tumor DNA in Late Stage Non-Small Cell Lung Cancer Patients. Genes 2019, 10, 926. [CrossRef]

(C) 2020 by the authors. Licensee MDPI, Basel, Switzerland. This article is an open access article distributed under the terms and conditions of the Creative Commons Attribution (CC BY) license (http://creativecommons.org/licenses/by/4.0/). 